\title{
2. Politique de la DDC et exemple d'une ONG
}

Brigit Hagmann, Kathryn Imboden, Philippe Berberat, Daniel Fino et François Mercier

\section{(2) OpenEdition}

\section{Journals}

Édition électronique

URL : http://journals.openedition.org/aspd/874

DOI : $10.4000 /$ aspd. 874

ISSN : 1663-9669

Éditeur

Institut de hautes études internationales et du développement

\section{Édition imprimée}

Date de publication : 1 avril 2001

Pagination : 53-83

ISSN : 1660-5934

\section{Référence électronique}

Brigit Hagmann, Kathryn Imboden, Philippe Berberat, Daniel Fino et François Mercier, «2. Politique de la DDC et exemple d'une ONG », Annuaire suisse de politique de développement [En ligne], 20 | 2001, mis en ligne le 27 août 2012, consulté le 08 septembre 2020. URL : http://journals.openedition.org/aspd/ 874 ; DOI : https://doi.org/10.4000/aspd.874 


\title{
2. POLITIQUE DE LA DDC ET EXEMPLE D'UNE ONG
}

\subsection{COMMENT LA DDC ENCOURAGE LE SECTEUR PRIVÉ}

\author{
Brigit Hagmann - Kathryn Imboden*
}

\section{INTRODUCTION}

Combattre efficacement la pauvreté suppose du travail, un revenu, la possibilité de constituer un pécule. La création d'emplois et la croissance économique sont l'affaire du secteur privé, tandis qu'il appartient à l'Etat d'assurer des conditions ambiantes favorables - par une politique économique appropriée, par une croissance axée sur la réduction de la pauvreté. La DDC s'emploie à promouvoir un développement qui profite aux couches sociales les plus démunies, en zone rurale comme dans les grandes agglomérations. Un tissu solide d'institutions publiques et privées est indispensable pour créer des emplois et assurer une certaine continuité. Les instruments d'une promotion efficace de l'emploi et des revenus portent notamment sur la production agricole et sa commercialisation, la promotion des petites entreprises, les services financiers, la formation professionnelle. Ce que la DDC en attend, c'est une évolution vers davantage d'équité sociale. Les programmes qu'elle mène dans ces domaines ont pour but d'offrir à son public cible - micro-entreprises, ménages, petits paysans - l'accès à des connaissances professionnelles et pratiques, à des moyens technologiques, à des services financiers, au marketing, etc. Pour ce faire, la DDC passe par des partenaires locaux qui sont bien implantés ou se montrent prometteurs. Elle les soutient dans leur développement tout en profitant elle-même de leur expérience.

Le présent chapitre décrit comment la DDC encourage le développement du secteur privé sous forme de promotion des petites entreprises dans les zones rurales et urbaines, quelle est la fonction de l'Etat dans ce domaine et à quels instruments on recourt. Puis nous examinerons dans quelle mesure ce mode de promotion du secteur privé contribue à diminuer la pauvreté. Ensuite nous passerons en revue les prestations prévues pour favoriser le développement des entreprises et les services financiers qui poursuivent le même but.

\section{POLITIQUE DE DÉVELOPPEMENT: PRINCIPES}

Il ressort du Rapport sur la politique suisse de coopération au développement 1976-1985 ${ }^{1}$ que pour la période en question, 33 des 39 projets de la DDC dont

* Brigit Hagmann: cheffe de la section emploi et revenus, DDC.

Kathryn Imboden : spécialiste du secteur financier, DDC.

La Section emploi et revenus fait partie de la Division ressources thématiques de la DDC. Elle est active dans les domaines suivants: promotion du secteur privé, agriculture et la commercialisation de ses produits, promotion économique, services financiers et formation professionnelle.

1. DDC, Rapport sur la politique suisse de coopération au développement 1976-1985, Berne 1987 (d, f, e). 
le volume financier dépassait le million de francs ont été réalisés par des partenaires étatiques ou para-étatiques. Depuis 1985, à côté d'instances gouvernementales, les partenaires importants de la DDC sont de plus en plus des acteurs de la société civile et des institutions ou organisations privées. L'Image directrice de la $\mathrm{DDC}^{2}$ spécifie entre autres: «Dans le cadre de l'exécution de notre mandat, nous soutenons des particuliers, des organisations non gouvernementales et des institutions publiques agissant au niveau local, national ou mondial. » Pour la DDC comme pour d'autres organisations donatrices, appuyer la société civile et encourager le secteur privé constituent de plus en plus un objectif important de la coopération. Son Image directrice est claire: «Nous soutenons les personnes défavorisées, afin qu'elles obtiennent un meilleur accès aux moyens de production et soient en mesure d'améliorer elles-mêmes leurs conditions d'existence.» La Stratégie 2010 de la $\mathrm{DDC}^{3}$ fournit des repères pour la mise en œuvre de l'Image directrice et pose un certain nombre de principes: les programmes de la DDC sont conçus pour obtenir le meilleur impact possible; ils favorisent les initiatives propres de ses partenaires afin de réduire la pauvreté, de s'attaquer aux causes structurelles des conflits, de soulager la détresse; dans un contexte international où les changements sont de plus en plus rapides, la DDC oriente son action en fonction des défis globaux. Par ailleurs, la DDC concentre ses activités sur cinq thèmes prioritaires: prévention et résolution des crises, bonne gestion des affaires publiques, promotion des revenus et de l'emploi, amélioration de l'équité sociale, utilisation durable des ressources naturelles. Et dans le cadre de la priorité "promotion des revenus et de l'emploi », la DDC combat le chômage et le sous-emploi en apportant son soutien au secteur privé par la promotion des petites entreprises rurales et urbaines.

Voici les objectifs globaux que vise la promotion des entreprises ${ }^{4}$ :

- stimuler une croissance qui réponde aux besoins de la population, qui permette d'offrir une gamme de biens et de services plus vaste et plus variée;

- créer les conditions nécessaires au maintien et à la promotion d'emplois stables et de revenus adaptés au travail fourni;

- tendre vers une répartition plus équilibrée du produit national au sein de la population et entre régions.

\section{$\square$ Chômage et sous-emploi}

Le manque de travail et de revenus est un des plus graves problèmes qui affectent les pays en développement aussi bien que les nations industrialisées. En effet, le travail constitue un élément décisif du développement économique et social. On estime qu'un tiers des trois milliards de personnes actives que compte la planète est sous-occupé ou au chômage. De plus, beaucoup de salariés - notamment dans les «pays de concentration» de la DDC - vivent sous la menace permanente de perdre leur emploi ; près de la moitié sont censés s'en tirer avec moins de deux dollars par jour. Plusieurs conférences des Nations Unies ont exhorté les gouvernements à combattre la pauvreté, et les pays participants ont convenu de réduire ce fléau de moitié à l'horizon 2015. Ce thème a

2. DDC, Image directrice de la DDC, Berne, 1999 (d, f, i, e, esp).

3. DDC, Stratégie 2010 de la DDC, Berne, 2000 (d, f, i, e, esp).

4. DDC, Politique sectorielle de la promotion de l'artisanat et de l'industrie, Berne, 1992 (d, f, e, esp). 
également été prioritaire lors du Sommet social de Genève en juin 2000 (Copenhague+5). La création d'emplois et de sources de revenus revêt une importance centrale à cet égard: créer du travail est l'affaire du secteur privé, tandis que le rôle de l'Etat consiste à mettre en place des conditions optimales. Le phénomène de la mondialisation provoque des transformations très rapides, d'où la nécessité d'une grande capacité d'adaptation de la part des acteurs économiques. Cette évolution met les travailleurs sous pression, les oblige à faire preuve d'une extrême souplesse, à profiter au maximum des possibilités de formation permanente. En zone rurale, des conditions ambiantes difficiles s'associent au manque de formation et à des services inadéquats pour compliquer encore plus l'adaptation des exploitations familiales. Les déséquilibres de la propriété foncière mettent une grande partie de la population dans l'impossibilité de couvrir ses besoins les plus élémentaires. Même si l'agriculture absorbe en principe beaucoup de main-d'œuvre, son potentiel est lui aussi limité. Le manque d'emplois dans les campagnes provoque l'exode rural qui reporte les problèmes dans les villes et leurs banlieues. Les secteurs modernes de l'industrie et des services se développent essentiellement au moyen de capitaux et sont, malgré leur croissance, faiblement créateurs d'emplois. De plus, les restructurations conduisent très souvent à la suppression d'activités traditionnelles. Les emplois du secteur public vont en diminuant, et c'est surtout du secteur privé que naissent de nouvelles activités économiques. Et ce sont toujours les emplois offerts par le secteur informel, les petites entreprises artisanales ou commerciales qui assurent la survie d'une grande partie de la population'.

\section{$\square$ Le rôle de l'Etat et celui du secteur privé}

Dans tous les programmes, il est primordial que l'on soit au clair sur les fonctions respectives du secteur public et du secteur privé. L'un et l'autre doivent être solidement organisés pour permettre la création d'emplois et leur maintien. Le moteur de la croissance économique et de l'emploi est le secteur privé, tandis qu'il incombe à l'Etat d'assurer des conditions ambiantes favorables, de pratiquer une saine politique économique et de veiller à une croissance socialement bénéfique. Sachant par expérience que les organes gouvernementaux manquent d'efficacité dans le domaine de la promotion économique, la DDC travaille de plus en plus avec des institutions et des centres de services privés. Elle ne participe pas directement au capital de sociétés privées, mais passe par des institutions d'encouragement. Ce faisant, la DDC veille à éviter que des fonds publics ne se substituent à du capital-risque privé.

\section{$\square$ Axer les efforts sur la lutte contre la pauvreté}

Encourager la création d'emplois et de revenus constitue un moyen nécessaire même s'il ne suffit pas à lui seul - de combattre la pauvreté. Il faut d'une part

5. A la veille du Sommet social de juin 2000 à Genève, le service sectoriel Industrie, formation professionnelle et développement urbain (intégré depuis octobre 2000 à la Section emploi et revenus) a organisé un colloque sur le thème «L'emploi - un outil contre la pauvreté». Le service sectoriel a publié pour cette rencontre une brochure portant le même titre, laquelle passe en revue les mesures que prend la DDC pour développer revenus et emploi. Ce document fait en outre une brève présentation de la situation internationale dans ce domaine. DDC, L'emploi - un outil contre la pauvreté, Berne, 2000 (d, f, i, e, esp). 
pouvoir offrir aux classes sociales défavorisées les moyens d'accéder aux emplois existants et à un revenu décent (p. ex. par la formation professionnelle), d'autre part stimuler une croissance économique visant à réduire la pauvreté par la création de nouveaux emplois rémunérés. Les pays donateurs sont aujourd'hui unanimes à penser qu'une croissance économique n'a pas automatiquement pour conséquence de diminuer la pauvreté (effet de retombée). Inversement, il ne faut pas croire que l'on peut créer du travail et des revenus - donc améliorer la situation sociale - sans croissance économique. Des synergies importantes peuvent naître entre production agricole et sa commercialisation, promotion des entreprises par des services financiers et autres, et formation professionnelle. Une première étape pour sortir de la pauvreté pourra être d'adhérer à un groupe d'épargne ou d'en fonder un, ce qui ouvrira par la suite l'accès à des crédits et à des possibilités d'investissement dont il résultera des emplois et des revenus. Ce genre de processus a lieu plus facilement dans les régions caractérisées par une bonne croissance de branches absorbant beaucoup de main-d'œuvre (surtout l'agriculture), ce qui suscite un nouveau potentiel avec des retombées positives sur le commerce. L'agent de ce développement est l'économie privée, laquelle dépend pour sa part d'un contexte favorable. Il est possible de stimuler le développement économique par des prestations ciblées - formation, management, commercialisation, technologie, conseil, financement - conçues en fonction du marché. Pour combattre utilement la pauvreté, il est important de rendre ces services peu coûteux et de les adapter aux besoins des microentreprises, des petits paysans, de toutes les personnes qui n'ont jamais bénéficié d'une formation ou d'un appui quelconque. Il convient de mettre au point en priorité des instruments qui impliquent des services accessibles aux plus démunis. Nombre des projets actuels de la DDC s'interrogent sur les mécanismes de promotion économique susceptibles de réduire la pauvreté. L'approche qui s'est révélée le plus clairement efficace jusqu'à présent consiste à intervenir au niveau "méso", en soutenant des business development services (BDS). Mais cette option ne convient pas toujours dans les zones de très grande pauvreté, lesquelles posent un défi dont il faut prendre conscience.

L'évolution rapide des sociétés actuelles requiert également une souplesse accrue de la formation, qui doit répondre à des exigences mouvantes. On y parvient en donnant à l'enseignement une structure modulaire, qui facilite l'accès aux étapes d'un apprentissage suivi d'une formation continue. C'est aussi le moyen de mieux adapter cette formation aux nécessités du travail et de la vie quotidienne. Cette approche moderne a aussi l'avantage d'exiger un comportement dynamique de la part des institutions publiques aussi bien que privées, chargées d'offrir des formations conformes aux besoins et à la demande.

La promotion des entreprises est un important moyen de combattre la pauvreté, en contribuant à créer et à perpétuer des emplois générateurs de revenus et de progrès social. Mais cette forme de promotion ne peut que soutenir des initiatives privées et doit exiger des efforts considérables de la part des bénéficiaires - qu'il s'agisse des institutions locales ou du destinataire final. La DDC entend éviter que le dynamisme potentiel de partenaires locaux soit étouffé par des apports extérieurs inconsidérés.

Le potentiel social de la promotion économique sera particulièrement favorable s'il y a des liens entre l'agriculture locale et des entités industrielles (axées sur 
l'exportation) relativement importantes. En zone rurale, cette promotion dépendra de certaines conditions générales: niveau relativement élevé des échanges monétaires dans la région, densité démographique suffisante, présence d'infrastructures de base (eau, électricité, transports, communications).

Il convient par ailleurs d'éliminer à divers niveaux les obstacles à une exploitation optimale des potentiels existants. Ces difficultés peuvent se résumer comme suit ${ }^{6}$ :

- conditions-cadres défavorables au développement; groupements d'intérêts/ organismes de promotion absents, inefficaces ou à la solde d'intérêts particuliers ;

- marchés d'approvisionnement et d'écoulement fragmentés, peu transparents, inaccessibles, absence de pouvoir d'achat;

- pas assez de personnes suffisamment qualifiées pour créer des entreprises et les gérer avec succès; productivité et qualité pâtissent du manque de professionnels expérimentés;

- équipements inexistants, vétustes ou mal adaptés aux conditions locales; accès difficile aux technologies et aux ressources financières.

\section{PROMOTION ÉCONOMIQUE PAR DES SERVICES DE DÉVELOPPEMENT D'ENTREPRISES (BUSINESS DEVELOPMENT SERVICES)}

\section{$\square$ Orientation stratégique et partenaires}

Les projets de développement institutionnel (mise en place, gestion et coordination d'organisations intermédiaires) et ceux qui visent à étoffer le marché des services ont pour but d'offrir aux PME, à des prix corrects, les prestations (business development services, $B D S$ ) dont celles-ci ont besoin pour se maintenir et se développer. Les partenaires de la DDC pour ces actions (au niveau «méso») sont essentiellement des organisations intermédiaires (business development centers) qui appuient sous une forme ou une autre des petites entreprises de tous secteurs. La DDC n'intervient que rarement - avec son personnel et des programmes de son cru - au niveau des entreprises individuelles, du fait qu'il serait trop long et coûteux d'acquérir à chaque fois les connaissances particulières à une branche donnée. Ses apports passent systématiquement par des partenaires compétents (organisations spécialisées, économie privée, organismes d'utilité publique) auxquels elle confie la responsabilité opérationnelle des programmes à mettre en œuvre. Elle trouve ses partenaires sur place ou ailleurs dans le monde. Ses interventions à l'échelon macro-économique ont pour but d'améliorer les conditions générales dans lesquelles travaillent les entreprises. Ce contexte a des aspects politiques, politico-économiques, juridiques et institutionnels - qui déterminent en partie le comportement des acteurs économiques sans que ceux-ci puissent exercer une influence directe sur ces facteurs. La DDC intervient généralement à ce niveau sous la forme d'accords internationaux, afin d'assurer que les mesures prévues soient largement approuvées et efficaces.

6. DDC, Politique sectorielle de la promotion de l'artisanat et de l'industrie, Berne, 1992 (d, f, e, esp). 


\section{Répartition géographique}

Tous les continents comptent des programmes consacrés à la promotion d'entreprises soutenus par la DDC. Une seule et même approche (BDS) est applicable en principe à n'importe quel pays bénéficiaire, mais il convient d'adapter les instruments de cette approche aux particularités économiques, politiques et sociales du pays en question. Des projets pour la commercialisation de produits agricoles ont été lancés en 2000 dans plusieurs régions du monde (Amérique latine, Europe de l'Est), et quantité de projets existants ont été infléchis dans le sens du marché. Le fait d'appliquer des nouvelles méthodes dans plusieurs régions simultanément permet d'encourager les échanges et l'accumulation des enseignements à différents niveaux. La DDC met à disposition quelque 25 millions de francs par année pour des projets de promotion d'entreprises.

\section{$\square$ Adaptation au marché plutôt que subventions}

La stratégie de la DDC en matière de promotion économique se base sur l'idée que des emplois durables ne sauraient se créer à coup de décrets gouvernementaux et dans le climat artificiel d'un programme de développement; ils doivent résulter de l'esprit d'entreprise et du potentiel engendré par des sociétés compétitives. Seule une entreprise viable peut assurer des emplois, raison pour laquelle la promotion économique ne doit pas concentrer son attention sur l'emploi sans se préoccuper de la viabilité de l'employeur.

En conséquence, la DDC estime que la promotion des entreprises doit découler d'une adaptation au marché et plus particulièrement d'une analyse de la demande et des potentiels existants. Cette analyse du marché permettra de définir à la fois des objectifs et une stratégie, ainsi que les ressources humaines, financières et techniques à prévoir pour un projet concret.

C'est le marché qui est déterminant pour l'existence d'une entreprise; celle-ci ne doit donc pas se construire et se maintenir en vie par des subventions. La DDC veille à éviter que ses apports ne procurent aux entreprises des avantages qui profitent directement à leurs prix de revient. Lorsque de l'argent provenant de la DDC est versé à des entreprises particulières, on s'assure que cette prestation fera l'objet d'une contrepartie appropriée ou qu'elle sera destinée à une catégorie spécifique (p. ex. des femmes). La DDC tient à empêcher que l'on privilégie des particuliers.

Des subsides peuvent être judicieux lorsqu'ils ont un rendement externe, notamment si leur utilité économique et sociale dépasse le coût de l'action considérée (p. ex. formation et conseil) ou s'il s'agit d'assurer l'accès de micro-entreprises ou de classes sociales démunies à certains services. La DDC n'accorde des subventions que si l'objectif, le montant, le déroulement de l'opération et sa durée sont clairement définis. On risque de créer des dépendances durables à l'égard de sources financières externes. Il convient de choisir les organismes de promotion en fonction de leur compétence professionnelle plutôt que sur la foi des programmes qu'ils présentent. Trop d'organisations privées s'en tiennent à un rôle caritatif et social plutôt que d'offrir des prestations qui correspondent aux besoins des entreprises et de la société civile.

L'exigence de professionnalisme et le principe de séparation des fonctions doivent conduire à une mise en œuvre coordonnée des instruments formation/ 
conseil d'une part, crédit/garantie d'autre part, mais en les confiant à des institutions différentes.

Les institutions qui ont donné la preuve de leur compétence professionnelle se voient attribuer de plus en plus des fonds destinés à des programmes plutôt qu'à des projets ponctuels, ce qui rend l'organisation encore plus performante. Cela signifie aussi que l'on remplacera une partie des contrôles d'exécution par l'examen des résultats en fonction des objectifs.

\section{$\square$ Promotion des femmes}

Tout projet de promotion économique doit comporter des aspects spécifiquement féminins afin de prévenir des discriminations, en procédant de manière différenciée selon le groupe cible. Lorsqu'il s'agit de micro-entreprises, la proportion de femmes est élevée, et les centres de services sont alors sensibilisés à des discriminations possibles. Tandis que des projets destinés aux PME doivent comporter, aux niveaux «micro» et «méso», des éléments qui permettent de cerner un public cible féminin et de lui offrir des prestations équivalentes (p. ex. fenêtres de crédit spéciales, produits et matériel didactique adaptés à des besoins spécifiques, prise en considération du temps disponible). Il faut également intégrer aux activités de ces projets des programmes d'information et de sensibilisation aux situations spécifiques des hommes et des femmes au sein de l'entreprise et dans la vie privée.

\section{$\square$ Technologie adaptée}

La DDC encourage l'utilisation de technologies adaptées, donc celles qui conviennent le mieux dans une situation concrète avec ses aspects économiques et socioculturels. La question de savoir s'il faut recourir à des procédés exigeants en capital ou en main-d'œuvre doit se traiter uniquement en fonction du marché. Et comme c'est au chef d'entreprise d'assumer le risque, soutien et conseils devront également lui servir à mieux évaluer diverses variantes et les risques qu'elles comportent.

\section{Conformité avec les nécessités sociales et écologiques}

La DDC veille au respect des principes locaux de politique sociale (p. ex. assurance sociale, lutte contre la discrimination des femmes, salaire minimum, interdiction d'exploiter des enfants, sécurité au travail). L'artisanat et l'industrie des pays en développement - surtout au niveau des petites entreprises - a souvent des effets nocifs sur l'être humain et l'environnement. Lorsque la DDC soutient un programme de promotion économique, elle contrôle que l'on suive les prescriptions nationales et internationales en matière d'environnement, encourage les améliorations techniques au niveau de la production et de l'élimination, appuie les actions destinées à faire prendre conscience des problèmes écologiques.

\section{Coopération multilatérale}

La DDC participe activement aux organismes internationaux de promotion économique pour que ses activités profitent des échanges de résultats scientifiques et d'expériences pratiques qui ont lieu à ce niveau, et aussi pour encourager la coordination entre donateurs d'une part et entre ceux-ci et pays en développement 
d'autre part. Le comité des donateurs pour la promotion des entreprises ${ }^{7}$ (qui existe depuis 1979) a publié en octobre 2000, après des années de coopération, des directives pour les interventions de promotion économique ${ }^{8}$. Ces directives sont basées sur la longue expérience et les «bonnes pratiques» de divers donateurs, et la DDC a participé activement à leur élaboration. Elles stipulent que pour être efficace, cette promotion doit s'inspirer d'emblée des besoins du marché et favoriser la création de services qui correspondent aux besoins des entreprises. Les subventions doivent être limitées en volume et en durée, et servir essentiellement à permettre aux créatrices et créateurs d'entreprises sans moyens d'accéder à des services.

\section{$\square$ Instruments}

La formation de base et la formation continue, en technique et en gestion, jouent un rôle essentiel dans la promotion économique. Formation commerciale et développement institutionnel revêtent une grande importance dès le moment où l'entreprise prend une certaine ampleur. D'excellents atouts techniques et une conjoncture saine ne servent à rien si le management n'est pas à la hauteur. La formation est dispensée par des établissements publics et privés, ou par des centres privés pour le développement des entreprises. La DDC cofinance parfois durablement des centres de formation professionnelle, ce qui leur permet de faire des offres plus avantageuses que les établissements privés. Il est toutefois important que les bénéficiaires (entreprises, personnes à former) doivent également payer pour une formation dont elles profitent de façon immédiate et directe. Les établissements de formation privés (business development centers) sont de leur côté contraints de pratiquer les prix du marché pour rester durablement viables. Il est aujourd'hui fréquent que des gouvernements ou des organisations donatrices mettent à disposition des bons couvrant une partie des frais de formation, afin de permettre aux personnes démunies d'accéder à ces offres. (Une étude en cours analyse les expériences faites avec des bons de formation en Asie et en Amérique latine ${ }^{9}$ ).

Le conseil individuel à l'entreprise est coûteux et ne s'applique aux micro- et petites entreprises que si le réseau de clients est suffisamment dense. Les conseillers sont des professionnels locaux (et non des experts internationaux), ce qui évite des obstacles linguistiques et culturels tout en réduisant les coûts. Des conseils de qualité requièrent des compétences à la fois techniques et pédagogiques. Les experts internationaux se consacrent presque exclusivement à la formation de conseillers locaux. Comme pour la formation, les clients sont invités à participer aux frais de conseil dès le début d'un projet. Cette participation a non seulement pour effet d'alléger le budget d'un programme de conseil, mais c'est aussi un indicateur de réussite simple et probant. Les conseillers sont en

7. DDC/Banque mondiale, Committee of Donor Agencies for Small Enterprise Development: What is it? What does it do? Berne 2000 (e).

8. World Bank/Committee of Donor Agencies for Small Enterprise Development, Business Development Services for SME's : Preliminary Guidelines for Donor-Funded Interventions, Washington 1998 (e). Les directives définitives ont été adoptées par ce comité en octobre 2000, et publiées début 2001 par la Banque mondiale.

9. Cette étude cofinancée par la DDC est réalisée par Swisscontact, Zurich. Elle pourra être obtenue auprès de la DDC ou de Swisscontact à partir du printemps 2001. 
outre plus motivés et plus attentifs à leurs clients lorsqu'ils doivent leur faire payer les prestations fournies, et les clients insistent davantage pour obtenir des conseils de qualité. Les centres de formation soumis aux lois du marché sont rarement en mesure d'offrir leurs conseils pour des prix qui soient à la fois rentables et accessibles aux petites entreprises.

L'encouragement de groupements d'intérêts constitue un instrument important de la promotion économique. Une association professionnelle aura toutes les chances d'être durable si elle présente des avantages manifestes pour ses adhérents. On recourt dans ce contexte à la dynamique de groupe pour identifier les intérêts communs et promouvoir la solidarité réciproque. Fonctions importantes des groupements d'intérêts: formation/conseil, normalisation, enregistrement et analyse d'indices propres à la branche, information sur les foires spécialisées, etc.

Dans les domaines qu'elle soutient, la DDC encourage la mise en place de réseaux qui servent à organiser les échanges d'informations, l'harmonisation des normes et des procédés, ainsi que la répartition du travail entre les diverses institutions. C'est plus particulièrement à l'échelon «méso» que la DDC s'emploie à renforcer les échanges de connaissances et d'expériences entre pays en développement, afin de stimuler les capacités locales.

Le dialogue politique avec des gouvernements peut aborder le sujet du développement des entreprises, lorsque les conditions-cadres font obstacle aux mesures de promotion tentées aux niveaux «micro» et «méso». Il s'agit de déterminer de cas en cas si l'on va faire dépendre le début ou la poursuite de l'activité dans un pays ou un projet de certains critères généraux (conditionnalité), ou si l'amélioration des conditions générales fera partie du projet en question.

Les décisions relatives aux contenus et aux méthodes à l'échelon «macro» se prennent en règle générale dans le cadre d'accords internationaux.

\section{$\square$ Mesure de l'impact}

Afin de connaître l'efficacité des projets de promotion économique au niveau des entreprises, la DDC travaille avec d'autres organisations donatrices nationales et internationales à l'élaboration d'un instrument destiné à mesurer l'impact, le champ d'action et la durabilité des interventions. Cet instrument doit non seulement servir de repère aux bailleurs de fonds, mais également permettre aux organisations intermédiaires et aux entreprises elles-mêmes de mesurer leurs progrès et leurs performances sur la base d'indicateurs simples. Il est difficile de collecter à peu de frais des données fiables dans le but de déterminer concrètement les effets que peuvent avoir des projets de promotion économique sur la création d'emplois et de revenus, donc sur la réduction de la pauvreté. Un groupe de travail international formé de membres du Comité des bailleurs de fonds pour le développement des petites entreprises, sous la direction de l'Organisation internationale du travail (OIT), est en train d'élaborer et de tester des indicateurs susceptibles d'être utilisés dans ces projets. Les premiers résultats sont attendus pour la fin de 2001 . 


\section{PROMOTION ÉCONOMIQUE PAR DES SERVICES FINANCIERS}

\section{$\square$ Deux axes stratégiques}

La DDC vise le développement durable d'institutions financières locales, bien placées pour répondre aux besoins d'une clientèle pauvre. Son approche consiste d'une part à rendre 1'outil «argent» accessible à des acteurs économiques sans ressources, jusque-là exclus du système financier conventionnel; d'autre part à soutenir le développement d'intermédiaires financiers viables à long terme.

\section{$\square$ Combattre la pauvreté en donnant accès à des services financiers}

Le public auquel la DDC s'adresse dans le domaine des services financiers est le même que dans d'autres secteurs où elle intervient: les populations défavorisées des pays où elle a choisi de concentrer ses efforts. Les programmes de soutien aux services financiers visent par définition des personnes particulièrement démunies. Par ailleurs, les services financiers prévus pour les PME ont également pour effet de créer des emplois et d'améliorer ainsi la situation économique de populations pauvres. Il y a toujours plus d'organismes financiers dont les services novateurs sont utiles à une clientèle jusqu'alors exclue de telles prestations. Et l'on admet aujourd'hui que l'accès même à des services financiers fiables est plus important que leur prix.

\section{$\square$ Miser sur le potentiel féminin}

Des services financiers particulièrement destinés à un public cible féminin ont un impact doublement bénéfique: lorsque le revenu des femmes augmente, il en résulte des progrès à la fois économiques et sociaux. Les femmes savent tirer parti de crédits appropriés pour créer des petites entreprises efficaces, tout en faisant profiter leurs familles (nutrition, santé, éducation) de ces activités. Il est avéré que les femmes se montrent d'une manière générale crédibles et fiables, qu'elles ont la réputation de respecter leurs engagements.

\section{$\square$ Soutenir des institutions financières viables}

On reconnaît qu'une offre accrue de services financiers durables doit passer par le renforcement d'institutions capables de les offrir, et non par le soutien à des actions ponctuelles dans le cadre des projets. Il s'agit donc de mettre en place des institutions locales durablement performantes, dont l'autonomie opérationnelle, puis financière, apportera la garantie de prestations adaptées aux besoins des populations visées.

La DDC a souscrit aux principes reconnus par l'ensemble des donateurs dans «Le financement des petites et micro-entreprises: principes directeurs pour choisir et appuyer les institutions intermédiaires $»^{10}$. Ces principes ont pour but d'établir des normes communes pour les donateurs qui souhaitent offrir aux petites entreprises la possibilité d'accéder à des services financiers. Leur cadre stratégique vise justement les deux objectifs complémentaires évoqués plus haut.

10. Document développé par le Comité des donateurs pour le développement des petites entreprises et le Groupe de travail des donateurs sur le développement du secteur financier, mars 1996. 
La DDC débourse plus de 10 millions de francs par an pour des programmes et projets touchant aux services financiers, notamment à la microfinance, sous forme de contributions généralement non remboursables. Elle octroie dans certains cas des prêts ou des garanties, et participe exceptionnellement au capital social. La DDC appuie également la formation, des ateliers d'échanges, des études et des initiatives de dialogue politique.

\section{$\square$ Les instruments de promotion}

La DDC met en œuvre - ponctuellement ou de façon combinée - des instruments de promotion adaptés à la situation initiale et aux conditions économiques et sociales qui règnent sur place. Ces instruments doivent respecter les principes généraux que s'est fixés la DDC tout en contribuant à

- soutenir les organisations partenaires sur les plans institutionnel, organisationnel et financier,

- identifier, tester, adapter et diffuser des technologies novatrices, propres à réduire les coûts côté demande aussi bien que côté offre,

- atténuer les risques.

\section{$\square$ Le développement institutionnel}

Les apports qui ont pour objet d'aider des institutions partenaires à se développer remplissent une fonction importante; ils revêtent souvent des formes variables au cours de la coopération. Ces contributions prennent appui sur les forces disponibles chez les partenaires eux-mêmes, et peuvent devenir très sélectives et ponctuelles au fil du temps. Les instruments auxquels on recourt dans ce domaine sont notamment le conseil en développement institutionnel, le suivi stratégique et technique, la formation et le perfectionnement de la direction, des cadres et du personnel. Elément essentiel: l'aide fournie par la DDC pour améliorer les instruments de contrôle et de gestion (p. ex. management information systems, $M I S$ ) et lors de l'informatisation (programmes d'équipement en matériel et en logiciels).

\section{$\square$ La participation au capital}

Compte tenu des risques prévisibles et de leur pondération, la DDC peut participer exceptionnellement au capital d'institutions financières au stade de leur création ou de leur développement ultérieur, dans le but d'en garantir une base de fonds propres qui leur permettra d'atteindre des objectifs importants sur le plan du développement. Ces apports de capital peuvent également servir à mobiliser des ressources locales supplémentaires.

\section{$\square$ Les prêts}

La DDC peut accorder des prêts lorsque cela s'avère utile pour surmonter une pénurie financière à moyen terme. Elle s'assure que l'on a d'abord examiné toutes les possibilités de financement à l'échelon local. Les conditions doivent être négociées de manière à éviter des distorsions du marché ou des situations préférentielles. La DDC prend en temps utile les mesures nécessaires pour assurer les remboursements et leur affectation. 


\section{$\square$ Les fonds de garantie}

Dans certaines conditions, des garanties peuvent faciliter l'octroi de crédits par les institutions financières. Mais elles sont rarement un moyen efficace d'assurer durablement l'accès du public cible à des crédits. La DDC peut prendre en charge les frais d'essai et de création d'instruments de garantie institutionnels, lorsque ceux-ci sont susceptibles d'éliminer des goulots d'étranglement dans l'accès au crédit.

\section{$\square$ Les fonds de crédit et de rotation}

Lorsque ces fonds existent, il convient d'œuvrer de sorte qu'ils soient gérés professionnellement - par du personnel compétent en matière de crédit et conformément aux critères en vigueur - et qu'ils débouchent, selon un calendrier rigoureux, sur des institutions appropriées. Là où l'on crée malgré tout un nouveau fonds, faute d'autres solutions, celui-ci doit se mettre en place dans la perspective d'une institutionnalisation prochaine par la politique du secteur financier.

\section{$\square$ L'établissement de contacts}

Des institutions appartenant au système financier peuvent également établir des contacts avec des organisations existantes d'auto-assistance, de clients ou d'intermédiaires (p. ex. ONG), pour réduire les frais de transaction et couvrir les risques (pression du groupe). La DDC peut fournir son aide pour l'identification de partenaires et la négociation de contrats de partenariat.

\section{$\square$ Les institutions de formation}

La DDC peut apporter son soutien à des établissements de formation extérieurs aux banques, à l'intention du personnel des institutions financières ou pour leur clientèle.

\section{$\square$ Les échanges de vues, la coopération, les études}

Il convient d'appuyer les réseaux nationaux, régionaux et internationaux, les séminaires et conférences, la préparation des échanges de vues et leur concrétisation. Le (co)financement ou la réalisation d'études sectorielles dans certains cas, spécifiques à un pays, peuvent revêtir une grande importance pour le développement de programmes ou de secteurs donnés.

\section{$\square$ Le dialogue politique et l'appui des réformes}

La DDC dialogue de façon régulière - autant que possible conjointement à d'autres bailleurs de fonds - avec le gouvernement du pays partenaire, et participe aux réformes de son secteur financier.

\section{$\square$ Répartition géographique}

La DDC soutient une série d'institutions situées en Afrique (notamment occidentale), dans le Sud asiatique, en Amérique latine, en Europe de l'Est et en exUnion soviétique (voir encadré), ainsi que le Women's World Banking, un réseau global d'institutions de microfinance dont la clientèle est formée de dirigeantes d'entreprises à faibles revenus. 
La DDC participe à des réseaux nationaux et internationaux, en particulier au "Groupe consultatif pour assister les plus pauvres», un consortium regroupant tous les donateurs bilatéraux et multilatéraux dans le domaine de la microfinance.

\section{CONCLUSIONS}

La promotion du secteur privé constitue un aspect important de la stratégie 2010 de la DDC, et les méthodes élaborées à cet effet depuis quelques années continueront d'être appliquées et affinées. Il s'agit avant tout d'examiner les options et les instruments utilisés en fonction de l'objectif principal de la DDC, qui est de combattre la pauvreté. La priorité est actuellement donnée aux instruments qui permettront aux groupes cibles de la DDC (les populations les plus pauvres) d'accéder à des produits et des services de première importance. Il n'est guère possible, à ce jour, de quantifier les résultats des programmes de promotion économique - raison pour laquelle la DDC tentera ces prochaines années, en étroite collaboration avec d'autres bailleurs de fonds et des ONG, d'élaborer des indicateurs applicables à tous les projets. Le travail effectué sur le projet measurement of job creation en collaboration avec d'autres donateurs et l'OIT devrait aboutir l'année prochaine à l'ébauche d'une méthode pour mesurer si des emplois se créent. Une tâche délicate sera de revoir les méthodes et les instruments qui ont fait leurs preuves dans les pays en développement pour les adapter aux situations qui règnent en Europe de l'Est et en Asie centrale. Les expériences faites dans ces pays seront rassemblées en collaboration avec la Division pour la coopération avec l'Europe de l'Est et la CEI, afin d'en tirer les éléments nécessaires à l'adaptation des stratégies de promotion du secteur privé.

\section{EXEMPLES"}

Inde - production de la soie

Lorsqu'une famille de petits paysans plante un hectare de mûriers, elle crée au total treize emplois dans la production de la soie, plus précisément pour la culture des mûriers, l'élevage des vers, le dévidage, le tordage, la teinture et le tissage de la soie. La culture de la soie en Inde, avec 312000 hectares de mûriers, offre ainsi du travail pour 4 millions de personnes. En comparaison, un hectare de canne à sucre crée 1,5 emploi.

Cela fait plus de vingt ans que la DDC encourage la sériciculture en Inde. Ce secteur constitue un important réservoir d'emplois et le marché de la soie est très vaste. C'est en particulier grâce au soutien de la DDC qu'un centre de formation - I'International Centre for Training and Research in Tropical Sericulture (ICTRETS) - a été mis sur pied. II s'y forme un grand nombre de spécialistes indiens et de divers pays d'Asie, d'Afrique et d'Amérique latine. Avec ses 14'000 tonnes de soie brute, I'Inde est le deuxième producteur mondial de soie, juste derrière la Chine. Aucun autre secteur d'exploitation agricole ne crée autant d'emplois.

La promotion de la production séricicole est une contribution importante au développement économique du secteur rural et paysan. Elle crée du travail pour les petits paysans et leur assure un revenu régulier. Plus de la moitié du travail d'élevage des vers est effectué par des femmes. Ce revenu améliore leur

11. Extraits de: DDC, L'emploi - un outil contre la pauvreté, Berne, 2000 (d, f, i, e, esp). 
situation économique, leur confère estime et respect au sein de leur famille et de la société, et leur permet de diriger elles-mêmes le cours de leur existence.

Les mûriers et les vers à soie sont des organismes vivants. Ni la culture ni l'élevage ne pourront être totalement industrialisés, de sorte que la fabrication de la soie restera tributaire du travail manuel et de petites structures artisanales. Cette production créatrice d'emplois permet aux petites entreprises de suivre durablement l'évolution technique et favorise l'initiative privée. Elle offre des possibilités de développement économique à plusieurs millions de personnes en Inde.

La DDC soutient la production de la soie à tous les échelons - depuis l'amélioration des semences jusqu'à la recherche de vers à soie plus résistants, en passant par le perfectionnement des méthodes de transformation de la soie brute et une productivité accrue des procédés de teinture ou de tissage. Elle accorde par ailleurs une grande attention aux questions écologiques et sociales: intégration des femmes, abolition du travail des enfants, amélioration des techniques de blanchiment et de teinture, optimisation des dépenses énergétiques.

Entre 1980 et 1994, la DDC a investi environ 5 millions de francs dans ce domaine. Elle a en outre consacré 4 millions à l'encouragement de la production de la soie en Andhra Pradesh et dans le Tamil Nadu. Et le cofinancement du "National Sericulture Project" de la Banque mondiale, entre 1989 et 1996, constitue jusqu'à présent le plus gros engagement de la DDC comme opération de cofinancement. Son but était d'améliorer qualité et rendement de la sériciculture dans cinq Etats de I'Union. Cet objectif a pu être atteint en améliorant les conditions de formation et la position des femmes dans le processus de production. La Banque mondiale et la Suisse ont investi respectivement 200 et 30 millions de francs dans ce projet.

\section{Roumanie - promotion des petites entreprises}

Le programme de promotion des petites entreprises en Roumanie vise à soutenir les réformes nécessaires pour y instaurer une économie de marché. Ses bénéficiaires sont des spécialistes roumains, motivés et compétents, qui ont ainsi la possibilité de lancer leur propre entreprise. La Suisse aide ces petites exploitations roumaines à obtenir des petits crédits et des machines appropriées, ainsi qu'en leur assurant une formation professionnelle et des notions de gestion d'entreprise. Les partenaires de ce projet en Roumanie sont les organisations d'utilité publique ROMCOM et l'association professionnelle Immanuel, qui regroupe de nombreuses petites entreprises. Depuis 1991, la Mission chrétienne pour les pays de I'Est a équipé environ 25 exploitations et assuré la formation des gens qui y travaillaient. Ces établissements partis de rien sont maintenant indépendants et emploient jusqu'à 12 collaborateurs et apprentis. Plus de 1400 emplois ont ainsi été créés. La DDC soutient ce projet depuis 1993.

Bénin - entraide des femmes

En juin 1995, il y avait à Cotonou, ville principale du Bénin, douze caisses d'épargne comprenant 1436 membres. Les dépôts s'élevaient à 7,1 millions de francs CFA (17'750 francs). Fin 1998, il y avait déjà 23 caisses d'épargne totalisant 4343 membres et 42,6 millions de francs CFA (106'500 francs). Deux à trois fois par semaine, une caisse d'épargne et de prêt s'ouvre à des emplacements inattendus - dans une école, un dispensaire ou parfois même sous un arbre.

Comme la plupart des villes africaines, Cotonou se développe rapidement; un quartier s'ajoute à l'autre, sans électricité ni eau courante. Presque personne n'a un emploi fixe, et c'est en général aux femmes qu'on laisse le soin d'assurer la survie de la famille. Elles se débrouillent pour apporter le minimum vital à leur famille en transformant des produits agricoles ou par des travaux de couture. Le modeste capital de départ que demandent ces petites entreprises ne peut que rarement être réuni en raison d'autres priorités - par exemple les soins à donner à des enfants malades. Cette situation a suscité en 1994 la création d'un système simple de crédit et d'épargne, organisé par quartiers et par les femmes elles-mêmes. 
La DDC, qui soutenait déjà un programme d'alphabétisation destiné aux femmes, s'est également investie dans ce projet pilote ("Association d'entraide des femmes"). Elle les a aidées à mettre sur pied leur organisation; les décisions stratégiques appartenaient aux femmes.

II a été clair dès le début que la DDC ne subventionnerait pas les crédits. Le personnel et les cadres ont suivi des cours de perfectionnement et acquis les connaissances nécessaires à la gestion de leur caisse d'épargne. Les caisses ont reçu le statut juridique de sociétés simples. Grâce à ces établissements microfinanciers, les femmes, jusque-là coupées de l'accès à des crédits, ont pu développer leurs activités professionnelles, améliorer leurs conditions de vie et défendre elles-mêmes leurs intérêts.

\section{Bolivie - réforme du secteur financier}

Un système bancaire efficace en zone rurale constitue un facteur essentiel de prospérité économique, surtout pour l'agriculture et l'artisanat. II contribue ainsi à la création d'emplois. En Bolivie, il s'agit d'amener les institutions financières existantes à mieux comprendre les besoins de leurs clients. Par exemple, elles n'offrent pas les services financiers que souhaiteraient les petites entreprises et les femmes de la campagne. La DDC a donc décidé de soutenir les établissements financiers existants (fonds financiers privés, ONG actives dans le domaine du crédit, petites coopératives d'épargne et de crédit) dans trois domaines: développement de nouveaux produits de microfinance (épargne, microcrédit, "leasing ", etc.), allocation plus efficace des crédits (réduction des frais de transaction) et formation du personnel. Ce programme compte comme partenaires pas moins de 75 fonds financiers et 30 ONG. II n'est pas possible de mesurer directement l'impact que peut avoir une telle intervention sur l'emploi et sur l'activité économique en général. Mais un contexte favorable aux activités économiques constitue la meilleure garantie de croissance, en particulier dans les zones rurales.

\section{Albanie - création d'associations d'épargne et de crédit}

La DDC contribue depuis 1992 au développement de services microfinanciers en Albanie, sous la forme d'un cofinancement avec la Banque mondiale (AID). Elle a soutenu dans un premier temps un programme de services financiers dans le district de Puka (nord de l'Albanie), en alimentant une ligne de crédit offerte aux fonds villageois de ce district par l'Albanian Development Fund. Ce fonds a été remplacé pour le crédit rural par un Rural Finance Fund; il s'agit là d'un organisme paragouvernemental destiné à soutenir la création d'associations d'épargne et de crédit, dans le but de privatiser ces activités pour en faire un réseau viable à long terme. La DDC finance des activités de formation et de développement durant la phase de transition vers un système autonome de ces associations.

\section{Women's World Banking}

La DDC finance également des initiatives globales, dont celle du Women's World Banking (WWB). Créée en 1979, cette institution financière à but non lucratif est destinée à promouvoir l'activité économique des femmes au niveau des micro- et petites entreprises. Avec ses 46 membres (affiliates) dans 39 pays, WWB est le premier réseau global dont la vocation est d'ouvrir aux femmes l'accès à des crédits. Ses clientes sont des dirigeantes d'entreprises industrielles, agricoles ou commerciales. Les membres du réseau sont des institutions financières locales qui fournissent des services financiers et logistiques aux entreprises. WWB New York est le pivot du réseau en termes de communication; ce centre fournit des appuis institutionnels, techniques et financiers, et constitue une plate-forme d'échanges et d'apprentissage collectif. Par ailleurs, WWB est à l'origine d'une évolution de la législation favorable au développement de la microfinance. 
Inde - BASIX, banque parallèle des zones rurales

BASIX est un nouveau type d'institutions destinées à développer des sources de revenus dans les zones rurales, créée en 1996 à Hyderabad, dans le sud de I'Inde. Sa mission est de fournir sous forme intégrée des services financiers et une assistance technique destinés à procurer des moyens d'existence à un grand nombre de personnes - en particulier aux pauvres et aux femmes de la campagne. BASIX vise des taux de rendement suffisamment intéressants pour accéder en permanence au marché des capitaux et aux ressources humaines. II regroupe des services financiers et des sociétés d'assistance technique. Bhartiya Samruddhi Finance Limited (Samruddhi), enregistrée auprès de la Reserve Bank of India en tant que Non Banking Finance Company Limited, est le principal opérateur en matière de crédit. Indian Grameen Services (IGS) est une ONG qui fournit de l'assistance technique et des services logistiques aux emprunteurs ainsi qu'à d'autres producteurs et organismes ruraux. Ces deux entités sont regroupées sous le holding BASIX Ltd. par lequel ont eu lieu les investissements initiaux dans Samruddhi.

BASIX a expérimenté divers produits financiers par plusieurs canaux à la fois, permettant ainsi à des groupes défavorisés d'accéder plus facilement à ce genre de services. Outre les prêts qu'elle octroie directement à ses clients, BASIX passe par des associations de production de semences, des agents de commercialisation de produits agricoles, des coopératives, etc. Ses représentants sur le terrain fournissent des services financiers à domicile. La clientèle active de BASIX est de dix mille personnes, et son portefeuille de prêts se chiffre à quelque 5,4 millions de francs suisses. Cette formule a également eu des incidences sur le système financier dans son ensemble. BASIX exerce une grande influence en matière de politique financière du fait qu'elle participe activement aux forums importants que sont par exemple Microfinance Task Force et Sa-Dhan, une association faîtière des institutions de microfinance en Inde. Elle fait en outre partie des premières sociétés financières non bancaires de I'Inde à s'engager dans le secteur de la microfinance. BASIX a par ailleurs aidé d'autres ONG à rationaliser leurs services financiers et autres. Enfin, la Reserve Bank of India a décidé de conférer à BASIX un label de qualité en lui accordant l'autorisation d'exercer des activités bancaires à l'échelon local, ce qui en fait une des trois premières banques locales en Inde.

La DDC a soutenu BASIX dès sa création par un apport financier et de l'assistance technique.

\section{SOURCES}

DDC, Politique sectorielle dans le domaine financier, Berne, 1998.

DDC, Politique sectorielle de la promotion de l'artisanat et de l'industrie, Berne, 1992 (d, f, e, esp).

DDC/Banque mondiale, Committee of Donor Agencies for Small Enterprise Development: What is it? What does it do? Berne, 2000 (e).

DDC, L'emploi - un outil contre la pauvreté, Berne, 2000 (d, f, i, e, esp).

DDC, Rapport sur la politique suisse de coopération au développement 1976-1985, Berne 1987 (d, f, e).

DDC, SDCs' CIP/SED Program in the Nineties: Review and Challenges ahead, Issue Paper no. 4, Bern, 1999 (e).

DDC, Image directrice de la DDC, Berne, 1999 (d, f, i, e, esp).

DDC, Stratégie 2010 de la DDC, Berne, 2000 (d, f, i, e, esp).

Banque mondiale/Committee of Donor Agencies for Small Enterprise Development, Business Development Services for SME's: Preliminary Guidelines for Donor-Funded Interventions, Washington, 1998 (e).

Banque mondiale/Committee of Donor Agencies for Small Enterprise Development and Donor's Working Group on Financial Sector Development: Micro and Small Enterprise Finance Guiding Principles for Selecting and Supporting Intermediaries, 1995 (e, f, esp). 


\title{
2.2. INSTRUMENT DE LA GARANTIE BANCAIRE INTERNATIONALE AU SERVICE DES PLUS PAUVRES
}

\author{
Philippe Berberat - Daniel Fino - François Mercier*
}

\section{INTRODUCTION}

Dans pratiquement tous les pays en voie de développement (PVD), la grande majorité des activités économiques se déroulent dans le secteur dit informel ou non structuré, aussi appelé le secteur de l'économie populaire. Les artisans, commerçants, producteurs agricoles, éleveurs, travaillant seuls ou en petits groupes, assurent l'essentiel de la production, la commercialisation et la distribution des biens de consommation de tous les jours. La principale caractéristique du secteur, qui est aussi son atout, est qu'il est composé de petites structures, voire «microstructures »: des ateliers très simples, des volumes d'affaires (par unité) faibles, des revenus et volumes d'affaires en moyenne plutôt modestes, un nombre limité d'emplois. Mais le secteur pose aussi des problèmes: outre le fait que le secteur échappe pratiquement à l'imposition fiscale, les revenus sont essentiellement utilisés pour la consommation et ne sont pas réinvestis, d'où un niveau d'équipement assez rudimentaire. Par un mode d'organisation informelle, des activités restreintes au marché local, une productivité relativement faible, etc., le secteur arrive difficilement à étendre ses activités (pour produire au moins pour un marché national, sans envisager forcément l'exportation), créer de la valeur ajoutée significative et faire face à la concurrence d'importations massives venant d'autres pays en voie de développement.

Un des blocages importants pour permettre à des acteurs économiques dynamiques du secteur informel d'investir, de développer des nouvelles activités et d'innover, est la difficulté d'avoir accès aux ressources financières nécessaires, en d'autres termes d'avoir accès au crédit.

Ces dernières années, le thème de la microfinance, c'est-à-dire la question de la mobilisation, de l'accessibilité et de l'utilisation de ressources financières pour l'économie populaire, est devenu d'une grande actualité. Il y a beaucoup de propositions et d'expériences qui sont discutées ${ }^{12}$ que nous ne pouvons pas aborder dans cet article.

Nous nous limitons ici à un instrument précis, très connu dans l'économie moderne, qui est la garantie $^{13}$. Dans quel cadre la garantie peut-elle avoir un

* Philippe Berberat: conseiller spécial, fondation Rafad, Genève.

Daniel Fino: chargé de cours et directeur adjoint du Service études et projets de l'IUED; président du Comité exécutif de la fondation Rafad, Genève.

François Mercier: adjoint de direction, fondation Rafad, Genève.

12. Les réflexions concernent les instruments à développer pour promouvoir les capacités financières des plus pauvres, les conditions à appliquer pour les institutions de microfinance (IMF), le rôle des banques alternatives, la recherche de formes d'assurances et de leasing pour le secteur de l'économie populaire, etc.

13. La forme la plus connue est le crédit hypothécaire, où un bien immobilier sert de garantie à la banque qui prête de l'argent au propriétaire. Un autre exemple, plus proche de la problématique qui nous intéresse, est un fonds de garantie créé entre les deux guerres, en Suisse, par les associations d'artisans avec l'aide de la Confédération pour cautionner des projets présentés aux banques. Le fonds existe toujours. 
rôle à jouer dans les PVD? Pour aborder cette question, nous sommes obligés d'étendre un peu la réflexion aux différentes situations possibles auxquelles les demandeurs de crédits se trouvent confrontés:

1. il n'existe pas d'institutions financières pouvant octroyer des ressources financières à des petits entrepreneurs du secteur informel ;

2. les banques modernes sur place traitent avec les acteurs du secteur moderne et ne s'ouvrent pas aux acteurs du secteur informel;

3. il y a des systèmes d'épargne et de crédit (caisses locales, coopératives d'épargne-crédit, mutuelles) qui peuvent octroyer des crédits;

4. des organisations de coopération internationale qui octroient des crédits subventionnés (à des conditions préférentielles);

5. il y a des formes traditionnelles (par exemple les tontines) qui mobilisent de l'épargne et la redistribuent;

6. les demandeurs de crédit s'adressent à des usuriers, avec tous les problèmes que cela comporte (coûts exorbitants)!

L'instrument de la garantie entre seulement en ligne de compte pour les situations 2 et 3 . Indépendamment du système bancaire (banque commerciale, banque coopérative, banque alternative, etc.), chaque institution prêtant de l'argent souhaite se couvrir par des garanties (des hypothèques ou d'autres formes de cautionnement). Cela pour éviter qu'en cas de non-remboursement du crédit, l'institution prêteuse subisse des pertes. Dans les PVD et notamment pour les acteurs de l'économie populaire, le manque de garantie constitue la principale barrière pour trouver le financement. Nous parlons ici de projets «bancables», qui remplissent donc tous les critères exigés. Il s'agit aussi de projets qui peuvent jouer un rôle important dans le développement socio-économique du pays car ils sont intéressants techniquement (ouverts à des innovations), économiquement (création de revenus) et socialement (création d'emplois).

Pour faciliter l'accès à des ressources financières pour des acteurs qui ne sont pas en mesure de fournir les garanties nécessaires, la création de fonds de garantie peut être une solution. Il y a différentes formes et type ${ }^{14}$ de fonds de garantie, mais le principe reste toujours le même: il y a un fonds (constitué de liquidités, de papiers de valeurs, de lettres de cautionnement de personnes physiques ou morales, etc.) qui est bloqué et qui sert à garantir des crédits accordés par une institution financière. L'utilisation d'une garantie constitue un coût qui doit être ajouté au coût du crédit.

Est-ce que l'outil de la garantie peut contribuer à résoudre le problème de l'accès au crédit pour des petits producteurs et commerçants de l'économie populaire des PVD? Pour répondre à cette question, nous allons montrer l'exemple d'une institution suisse, Rafad ${ }^{15}$, qui travaille depuis quinze ans avec cet outil.

14. Des fonds déposés auprès d'une banque dans le pays où les prêts sont octroyés, des fonds situés à l'extérieur du pays, des fonds gérés par les institutions prêteuses elles-mêmes, des fonds gérés par des organisations indépendantes, etc. Les fonds de garantie qui interviennent dans les PVD sont en règle générale soutenus par la coopération internationale, notamment pour couvrir les frais de fonctionnement.

15. Recherches et applications de financements alternatifs au Développement, fondation de droit suisse, créée en 1985 par Charles A. Egger, Bernard Faure, Mahamian Kipiangah, Bernard Lecomte, Fernand Vincent. 
L'approche sera illustrée par deux études de cas. Nous terminerons notre article par une réflexion plus large sur la garantie car l'utilisation de cet outil pour les PVD est controversée.

\section{L'ORIGINE DE RAFAD ET SON ÉVOLUTION}

Rafad a été créé il y a une quinzaine d'années. Ses fondateurs poursuivaient des objectifs de différentes natures mais tous innovateurs et ambitieux. Notons parmi les plus importants :

- Le changement des relations d'aide pour diminuer le rôle du don dans la coopération internationale qui ne favorise pas 1'autopromotion et pour faire jouer au système d'aide un rôle plus indirect et modeste d'appui aux dynamiques internes dans les pays.

- L'introduction d'instruments financiers de l'économie moderne pour le secteur de l'économie populaire.

- La mobilisation des ressources financières localement disponibles (épargne nationale) en faveur des organisations locales en leur ouvrant l'accès au crédit.

- Le renforcement des compétences de celles-ci pour gérer des fonds (par la formation, l'accompagnement).

- La recherche appliquée et la réflexion sur les questions de financement du développement (par des études, la diffusion des expériences, l'information).

Rafad a pu commencer à octroyer des garanties grâce à des ressources que l'un des membres fondateurs avait mis à disposition, ainsi qu'à la mise à disposition d'une contre-garantie ${ }^{16}$ de la Confédération. La fondation est depuis sa création en contact étroit avec un important réseau de partenaires: des ONG nationales, des associations, des caisses d'épargne-crédit, des groupements de base, etc. Pour travailler en proximité avec le terrain et pour s'assurer d'une plus grande objectivité dans l'appréciation des dossiers, Rafad dispose de consultants locaux qui travaillent, avec des contrats de prestation, dans les pays où des garanties sont émises. Ils assurent le lien avec le bureau à Genève et traitent avec les partenaires et les associations de base pour réunir les informations nécessaires, compléter les dossiers de demandes de garantie, étudier et apprécier les données fournies, négocier avec la banque, assurer le suivi, etc.

Afin de pérenniser l'expérience de Rafad en faisant évoluer la structure qui octroie des garanties vers une société contrôlée par les bénéficiaires des garanties, en 1996, la coopérative Fonds international de garantie (FIG) a été créée. Rafad se trouve actuellement dans la phase de transfert de toutes ses activités de garantie vers le FIG, ce qui aura aussi l'avantage de mieux séparer les activités de recherches et de consultations du système de garantie. Le FIG, géré par Rafad et qui prend sa relève, travaille dans le même créneau où Rafad a travaillé jusqu'à ce jour. La différence significative est que seuls les associés de la coopérative FIG peuvent bénéficier d'une garantie et que la part sociale des associés est mise à contribution en cas d'appel de leur garantie. Pour bénéficier d'une garantie, il faut donc obligatoirement être associé du FIG et donc avoir acheté des parts sociales du FIG.

16. C'est une lettre dans laquelle la Confédération s'engage à honorer des éventuelles pertes qui interviendraient suite à l'émission de garanties octroyées par Rafad. La lettre est déposée auprès de la banque qui exécute les ordres d'émission de Rafad. 


\section{Quelques données sur Rafad/FIG}

Taille du fonds de garantie CHF 3'960'228.- (dont Rafad: 3'506'158, FIG : 454'070)

\begin{tabular}{|c|c|}
\hline Nombre d'associés (FIG) & 23 personnes et organisations \\
\hline Garanties émises & CHF 1'749'668.- (dont Rafad: 1'575'248.-, FIG 174'420.-) \\
\hline Nombre de dossiers & $\begin{array}{l}18 \text { (dont Rafad: } 15, \mathrm{FIG}: 3 \text { ) } \\
\text { Dossiers à l'étude : } 6\end{array}$ \\
\hline Représentation sur le terrain & Consultants locaux dans 10 pays (dont 7 pays avec des projets en cours) \\
\hline $\begin{array}{l}\text { Répartition géographique } \\
\text { des garanties }\end{array}$ & $\begin{array}{l}\text { Amérique du Sud: } 58 \% \\
\text { Amérique centrale: } 4 \% \\
\text { Afrique: } 38 \%\end{array}$ \\
\hline $\begin{array}{l}\text { Montant moyen } \\
\text { d'une garantie }\end{array}$ & CHF 97'000.- (54'000 dollars) \\
\hline \multirow[t]{2}{*}{$\begin{array}{l}\text { Profil des } \\
\text { organisations partenaires }\end{array}$} & $\begin{array}{l}\text { - Clients directs: } \\
\text { - Organisations d'appui aux micro-, petites et moyennes entreprises } \\
\text { (43\% du portefeuille) } \\
\text { - Organisations d'épargne-crédit et institutions de microfinance }(14 \%) \\
\text { - Organisations paysannes }(43 \%)\end{array}$ \\
\hline & $\begin{array}{l}\text { Clients indirects: } \\
\text { Micro-, petites et moyennes entreprises du secteur agricole, artisanal } \\
\text { et commercial actives sur les marchés internationaux et locaux }\end{array}$ \\
\hline
\end{tabular}

Avant l'octroi d'une garantie, le plus important du travail consiste à évaluer le risque. Différents facteurs entrent en ligne de compte pour l'évaluation du risque, comme:

- le risque institutionnel (la «qualité» du partenaire, sa capacité de gestion et de suivi, la volonté de transparence, l'expérience dans le domaine, etc.);

- le risque économique (la faisabilité du projet, sa rentabilité, le taux de remboursement des crédits, etc.);

- le risque financier (capitalisation, solidité financière de l'ONG);

- le risque pays (contexte politique, financier, socio-économique, législation locale, etc.);

- le risque banque (collaboration de la banque locale);

- autres risques (transport, corruption, etc.).

Entre 1985 et septembre 2000, le taux de pertes global de Rafad/FIG se situe en moyenne à $5.6 \%$ par an des garanties émises.

Un deuxième aspect important à étudier avant l'octroi de la garantie est l'effet de levier attendu, c'est-à-dire le montant qui sera finalement mis en circulation dans le circuit économique grâce à l'octroi de la garantie. En moyenne, l'effet de levier chez Rafad est de plus de 3, c'est-à-dire que pour 1 franc de garantie, 3 francs de crédit ont été accordés. 
Un troisième élément qui entre dans l'appréciation du dossier est l'impact $d u$ projet sur la réduction de la pauvreté. Combien d'emplois pourront êtres créés par le crédit octroyé grâce à la garantie? Quels revenus et épargnes seront engendrés? Comment la communauté locale pourra-t-elle en bénéficier?

Nous savons que des milliers de personnes ont pu trouver du travail et améliorer leur revenu, mais l'impact sur la réduction de la pauvreté est difficile à évaluer. Rafad dispose d'un certain nombre de données que nous retrouvons dans les exemples ci-après, mais aussi dans des évaluations qui ont été faites (août 1997).

La garantie n'est bien sûr pas le seul outil pour promouvoir les activités économiques à la base, mais dans des situations spécifiques, elle peut être un instrument adapté, voire incontournable, pour permettre l'accès à des ressources financières, localement disponibles, à des opérateurs de l'économie populaire. Les études de cas résumées ci-après donnent un petit aperçu de la variété de situations dans laquelle la garantie peut jouer un rôle.

\section{DEUX ÉTUDES DE CAS AVEC LA GARANTIE DU SYSTĖME RAFAD}

\section{$\square$ Le partenariat de Rafad}

avec le réseau d'épargne-crédit Nyesigiso au Mali

Au Mali, l'un des pays les plus pauvres du monde, un formidable réseau de caisses d'épargne-crédit locales s'est formé depuis 1989. Initié et soutenu par une ONG canadienne, Nyesigiso a également pu compter sur l'aide de Rafad dès 1997 par le biais d'une garantie bancaire. Dix ans après sa création, le réseau ne compte pas moins de 44 caisses et près de 50'000 membres. En 1999, Nyesigiso a pu se passer du concours de Rafad et a retourné la garantie.

\section{Une situation économique paradoxale}

La situation macro-économique du Mali au milieu des années 1990 présentait de très lourds handicaps, auquel s'ajoute le fait que le secteur de l'économie informelle représentait $80 \%$ à $90 \%$ de l'économie du pays. Dans ces conditions, l'obtention de crédits auprès des institutions financières maliennes pour le secteur informel était extrêmement difficile, alors même que les banques commerciales disposaient de surliquidités importantes.

\section{Un réseau basé sur la confiance}

Travaillant exclusivement avec le secteur informel, Nyesigiso se compose actuellement de caisses locales indépendantes qui recueillent l'épargne et distribuent des crédits. L'épargne a la particularité de ne pas être rémunérée, car c'est le sentiment de sécurité qui représente la principale motivation d'adhésion à la caisse. En ce qui concerne le crédit, il n'est accordé qu'aux membres de la caisse et à de strictes conditions ainsi qu'avec des garanties (matérielles ou cautionnement). Basé sur la confiance, le système enregistre un taux de remboursement très élevé car il existe une forte pression sociale sur les mauvais payeurs. Les sommes prêtées sont plafonnées à FCFA 500'000 (CHF 1'250) pour les prêts individuels et FCFA 700'000 (CHF 1'750) pour les groupements, alors que la durée est inférieure ou égale à douze mois, pour un taux d'intérêt légèrement supérieur aux banques commerciales (12\% par an).

On peut encore souligner que Nyesigiso montre de bons états financiers. Le réseau a en effet réalisé d'importants bénéfices en 1996 et 1997, il dispose de 
suffisamment de fonds propres et n'a pratiquement pas de charges financières puisque l'épargne n'est pas rémunérée.

Une garantie Rafad destinée à renforcer sa capacité d'octroi de crédit

Dans le cas de Nyesigiso, l'argument qui a incité le réseau à se tourner vers Rafad était que l'un des partenaires du réseau (la Caisse des entrepreneurs) connaissait alors un essor considérable en matière de demandes de crédits, mais se heurtait à deux obstacles. Premièrement, il ne disposait pas du capital suffisant pour octroyer ses propres crédits, de plus, une des règles de Nyesigiso interdisait d'employer à son profit plus qu'un certain pourcentage de l'épargne recueillie par d'autres caisses.

La solution d'une garantie bancaire a donc permis d'avoir accès à un prêt extérieur au réseau et de répondre ainsi à la forte demande de crédits. La garantie Rafad a également permis de jouer le rôle de médiateur en mettant en relation le réseau Nyesigiso avec la banque locale.

\section{Tableau d'indicateurs}

\section{Garantie RAFAD:}

$\begin{array}{ll}\text { Emission } & 05.05 .1997 \\ \text { Annulation } & 19.10 .1999 \\ \text { Montant } & \text { CHF 150'000.- } \\ \text { Banque locale } & \text { Banque Internationale pour le Mali (BIM) - Bamako } \\ \text { Utilisation garantie } & \text { Risque de défaut du réseau d'épargne crédit } \\ \text { Effet de levier } & 3.3 \mathrm{x} \\ \text { Couverture du risque } & \square 70 \% \text { par les membres des caisses et la BIM, } \\ & \square 30 \% \text { par Rafad, dès le premier franc }\end{array}$

Impact social:

Nombre de personnes

a Le réseau comptait près de 50'000 membres en 1999.

a De plus, Nyesigiso a accordé 5828 crédits en 1997, crédits qui pour la plupart sont octroyés à des micro-entreprises familiales.

๑ On peut penser que Nyesigiso a vraisemblablement maintenu un nombre important d'emplois dans la population locale.

Nombre de femmes Proportion très importante de sa clientèle majoritaire en ce qui concerne les concernées crédits auprès des groupements. De plus, des programmes spécifiques pour l'aide aux femmes rurales démunies ont été mis en place. Enfin, c'est une femme qui dirige le réseau.

Politique sociale Les membres du réseau participent à l'orientation et au contrôle des caisses du partenaire de manière démocratique (une voix par membre).

Effet de formation Le réseau offre un appui technique et financier à chaque caisse locale.

Une banque locale qui trouve son intérêt dans le développement de son pays L'institution financière locale, la Banque Internationale pour le Mali (BIM), s'est fortement impliquée dans cette opération. En effet, suite à des négociations entre le réseau, Rafad et la BIM, celle-ci a couvert dès le début $50 \%$ des risques. La garantie Rafad a donc permis de générer un effet de levier de 2, qui a même augmenté ensuite à 3,3. 
Cet exemple de garantie a montré que deux systèmes bancaires ayant des logiques et des intérêts différents (Nyesigiso qui veut augmenter ses capacités d'octroi de crédit et la BIM qui veut utiliser ses surliquidités) peuvent parfaitement collaborer et contribuer au développement socio-économique du pays.

L'effet de la garantie a pleinement fonctionné, la banque et le réseau Nyesigiso se considèrent aujourd'hui comme des partenaires commerciaux. L'utilisation de la garantie a ensuite été orientée de manière à couvrir les risques de pertes éventuelles ou les manques de liquidités dues aux variations saisonnières.

La situation financière s'étant consolidée avec une marge brute couvrant tous les frais de fonctionnement sans subventions, l'utilité d'une garantie Rafad ne s'est plus justifiée. C'est la raison pour laquelle Nyesigiso a décidé de l'annuler huit mois avant son échéance.

\section{- Le système Rafad/FIG en faveur des producteurs de café du Pérou}

$\mathrm{Au}$ Pérou, depuis près de dix ans, la fondation Rafad entretient un partenariat avec la Coopérative agricole de café (CAC) la Florida. Celle-ci est la dernière coopérative de producteurs de café de la province de Chanchamayo et exporte plus du tiers de sa production vers le marché lié au commerce alternatif occidental. Avec les garanties de Rafad et du FIG, la coopérative finance le fonds de commercialisation pour l'achat du café à ses membres.

\section{Une farouche volonté de se reconstruire après de longues années d'insécurité}

Dans la province de Chanchamayo, région d'implantation de la Florida, la population a beaucoup souffert des massacres perpétrés par le Sentier lumineux au début des années 1990. S'ajoutant à la problématique du terrorisme, la présence, ces dernières années, de narcotrafiquants est venue amplifier le sentiment d'insécurité qui régnait dans la région.

C'est paradoxalement dans ce contexte particulier de grande insécurité que la coopérative a trouvé un deuxième souffle. Créée en 1966, elle était prospère et active, mais au début des années 1990, au plus fort de l'activité terroriste, la Florida ne comptait plus qu'une quarantaine de membres. De plus, à cette époque, suite à la libéralisation de la commercialisation du café, la coopérative se retrouvait sans le soutien qu'apportait traditionnellement l'Etat aux coopératives.

\section{Une activité à nouveau en pleine croissance}

En dépit de ces conditions, la volonté et le travail d'un groupe de membres particulièrement actif ont permis à la coopérative de se relever. Elle compte désormais quelque 700 membres actifs, accroît sa production chaque année et, bien qu'elle ne soit pas encore financièrement autonome, elle dégage néanmoins des bénéfices de l'exportation du café. La Florida tire aussi son épingle du jeu en se concentrant sur une production de grande qualité qu'elle écoule ensuite en partie $(30 \%)$ vers le marché du commerce équitable occidental. Ces débouchés lui permettent de pouvoir compter sur des prix d'achat supérieurs à la moyenne.

Si la Florida a réussi à se redresser, il faut aussi souligner l'importance de l'aide qu'elle a reçue de la part d'associations du Nord. Des acteurs traditionnels du «Fair Trade» comme ROTHFOS (Allemagne), mais aussi Terre des hommes (Genève) et SOS-Faim (Belgique) l'ont ainsi soutenue financièrement. La très active organisation genevoise La Florida-Pérou lui offre également un appui important pour ses activités sociales et de commercialisation. 


\section{Tableau d'indicateurs}

Garantie RAFAD:

$\begin{array}{ll}\text { Emission } & 15.05 .2000^{*} \\ \text { Annulation } & 31.12 .2000 \\ \text { Montant } & 125^{\prime} 000 \text { dollars } \\ \text { Banque locale } & \text { Banco Continental - Lima } \\ \text { Utilisation garantie } & \text { Approvisionnement et commercialisation de café } \\ \text { Effet de levier } & 1.4 \mathrm{x} \\ \text { Couverture du risque } & \square 72 \% \text { par Rafad (contre-garantie de la DDC) et le FIG } \\ & \text { (dont 4\% de parts FIG de la Florida) } \\ & \square 28 \% \text { par le Banco Continental dès le premier franc }\end{array}$

\section{Impact social:}

Nombre de personnes

La coopérative regroupe environ 700 producteurs de café. Elle crée plus de 2500 emplois directs et en maintient 450 indirectement grâce aux financements obtenus.

Nombre de femmes La Florida a mis sur pied deux "comités de femmes", d'environ 80 membres, concernées qui financent leurs activités grâce à des pépinières de café.

Politique sociale du partenaire

La coopérative octroie à ses membres des services de santé, d'éducation et de conseil juridique. Elle travaille également sous mandat afin de conduire les démarches pour la construction de routes, de ponts, de réseaux électriques et pour des services d'éducation.

Effet de formation Une école de formation dispense des cours de production et d'exploitation des terres avec des critères de qualité totale. De plus, une assistance technique octroyée par du personnel spécialisé accompagne le fonds rotatif destiné aux producteurs.

* Première garantie émise le 02.07.1990.

Un projet permettant d'améliorer les conditions de vie de la communauté

La Florida a mis sur pied un certain nombre d'infrastructures sociales, notamment au niveau de la formation, de la vulgarisation agricole, de la santé et de l'éducation. Au-delà de la place majeure qu'elle occupe sur le marché local du café, en particulier pour ce qui est de la fixation des prix, la Florida apporte une vision globale du développement. Elle contribue par là même à instaurer un climat propice à la stabilité et à la prospérité pour les 10'000 familles de la région.

\section{Le FIG, un outil financier adapté aux besoins de la Florida}

La nécessité de se tourner vers une aide extérieure s'est faite particulièrement pressante à partir de 1990. Privée de l'appui étatique, minée par les activités terroristes, la Florida devait impérativement dénicher un financement afin d'assurer un fonds de commercialisation pour l'achat de café à ses associés. L'accès au crédit local lui étant refusé sans l'apport de garanties, la coopérative s'est alors adressée à Rafad. Une première garantie a été émise pour la récolte de 1990 et a été renouvelée chaque année depuis lors.

En l'an 2000, la Florida est devenue membre du FIG (Fonds international de garantie) et a ainsi rejoint cette coopérative d'associés du Sud et du Nord dont le but est d'octroyer des garanties pour des partenaires au Sud. 
La baisse des prix du café a provoqué un appel de garantie, sans pourtant impliquer la faillite de l'organisation

La baisse du prix du café pose des difficultés pour la rentabilité de la coopérative. En 1992, la chute des cours du café a entraîné deux appels sur les garanties pour un total de CHF 50'000. La confiance en la volonté et les capacités de la Florida à vouloir se redresser n'a jamais quitté Rafad. La Florida a montré qu'elle était en mesure de tenir ses engagements en remboursant régulièrement les dettes issues de ces appels. A ce jour, son niveau d'endettement auprès de bailleurs de fonds étrangers est encore important, mais la coopérative a réussi à reprogrammer dans le temps ses dettes les plus importantes. La question des prix internationaux du café, sur lesquels la coopérative n'a pas d'influence, reste cependant d'actualité et est un souci majeur pour cette organisation.

\section{QUEL BILAN DE L'EXPÉRIENCE RAFAD/FIG?}

L'expérience de Rafad et du FIG permet de dresser un bilan intermédiaire de l'utilisation de la garantie. Elle permet de mieux situer l'instrument de la garantie bancaire avec ses succès, ses échecs, ses potentialités et ses obstacles.

Comme principal succès, il faut souligner la mobilisation et l'augmentation de ressources financières pour le secteur de l'économie populaire. Les pertes sont relativement faibles et l'effet de levier acceptable. Il convient aussi de souligner que la garantie permet de rapprocher les acteurs de l'économie populaire du secteur bancaire moderne.

Les difficultés majeures que rencontre l'utilisation de la garantie se situent au niveau des facteurs contextuels (situation économique morose, taux d'intérêts élevés, méfiance des institutions financières classiques, présence de prêts à des taux subventionnés, etc.).

\section{Schéma: Fonctionnement du FIG}

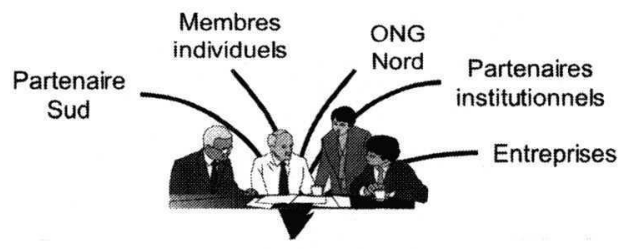

Fonds International de Garanties

Parts de CHF 1'200.-

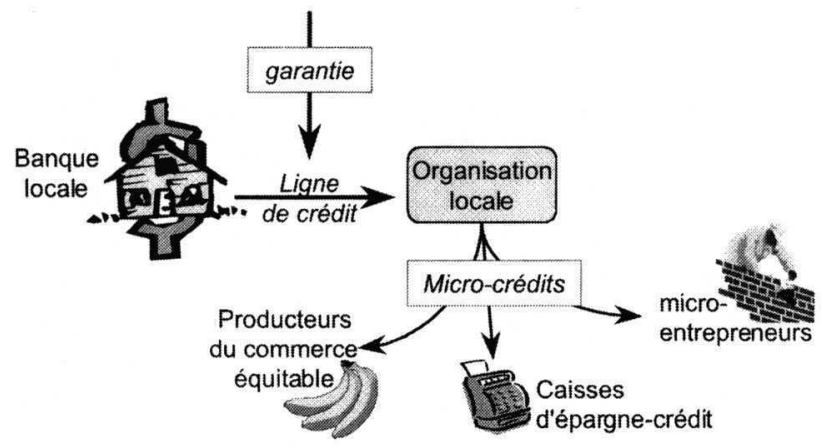


Un autre grand problème constitue le coût de fonctionnement d'un fonds de garantie international pour couvrir les frais liés à l'étude des dossiers, le suivi sur le terrain, la formation, les relations publiques, etc. Pour atteindre l'autofinancement du fonds et si on veut facturer une commission supportable et raisonnable (entre 2,5 et $5 \%$ du montant de la garantie) pour l'utilisateur de la garantie, le fonds doit atteindre un volume relativement important (entre 4 et 6 millions de $\mathrm{CHF}$ ), dont 60 à $70 \%$ doivent être engagés en émission de garanties. Ce montant n'est actuellement pas encore atteint par Rafad/FIG, ce qui rend le système encore dépendant du système d'aide.

Les potentialités résident avant tout dans le développement du FIG. Son avantage, comparé au système initial, est le fait que pour obtenir une garantie, le bénéficiaire doit être membre de la coopérative FIG, donc avoir acheté des parts sociales. Ceci devrait augmenter considérablement la responsabilité et la participation des membres et donc contribuer à ce que le FIG devienne une institution où les partenaires du Sud participent activement aux décisions. Par la forme juridique de la coopérative, qui fonctionne selon le système «un membre - une voix», il devrait être possible de gérer d'une manière démocratique le FIG, indépendamment du montant des parts sociales possédées par les associés.

Un autre avantage du FIG est la meilleure répartition du risque à l'intérieur de l'institution qui est couvert, progressivement, par les parts sociales du bénéficiaire de la garantie (qui est aussi associé), les fonds de réserve (provenant de dons) et finalement le capital social engageant l'ensemble des parts des associés.

Dans une perspective plus large, le FIG peut aussi être un instrument intéressant pour des organisations de développement au Nord qui peuvent

- devenir membres du FIG et ainsi effectuer des placements «utiles et solidaires »;

- acheter des parts pour faire bénéficier leurs partenaires du Sud en leur ouvrant un «guichet» garantie;

- participer à l'achat de parts pour des partenaires qui désirent devenir membres du FIG mais qui ne disposent pas des fonds propres suffisants (parrainage Nord-Sud).

Le FIG donne aussi la possibilité à des épargnants qui ne seront jamais demandeurs d'une garantie d'acheter des parts (non rémunérées, mais récupérables) et de renforcer ainsi la capacité financière du FIG.

Comme principal obstacle au développement du système, la détérioration des contextes socio-économiques, notamment les crises financières nationales et internationales, peut intervenir. La politique des agences d'aide internationale, qui devraient intervenir avec un grand doigté dans le secteur de l'économie populaire en utilisant les dons et les crédits subventionnés uniquement pour des situations sociales bien particulières, peut constituer un autre frein au développement du système.

Sur le plan local, il faut que le cadre propice (légal, institutionnel,...) soit donné pour permettre aux acteurs locaux d'émerger et de pouvoir développer leurs activités avec la plus grande sécurité possible. Le principal défi pour le FIG consiste alors à identifier des projets économiques situés au niveau de l'économie populaire qui, grâce à la garantie et au crédit, produisent une valeur ajoutée 
importante en termes économique, financier et social. Ceci dans le but de contribuer à réduire la pauvreté dans le pays.

\section{QUELQUES ÉLÉMENTS DE CONCLUSION: LA GARANTIE BANCAIRE EST-ELLE UN OUTIL POUR PROMOUVOIR LA MICROFINANCE?}

Cette question fait l'objet d'un large débat avec plusieurs études publiées ces dernières années ${ }^{17}$ qui démontrent fondamentalement deux tendances. D'un côté, les adversaires de la garantie bancaire estiment qu'elle ne crée pas d'avantages, que les systèmes de garantie ne sont pas financièrement autonomes à long terme et qu'il vaudrait mieux changer les réglementations bancaires de telle façon que les coûts de transaction et le crédit aux micros- et petites entreprises soient baissés. De l'autre côté, les supporters des systèmes de garantie considèrent que, sous certaines conditions, ils peuvent représenter un outil de promotion de la microfinance.

Parallèlement à ce débat, des banques commerciales dans les pays en voie de développement commencent de manière surprenante à s'intéresser au secteur de la microfinance, alors que jusqu'à présent, elles étaient réticentes vu le facteur «risque», les coûts élevés des transactions et le manque de nantissements adéquats. Des banques en Asie (certaines très proches des ONG) ont réalisé dans ce secteur, au cours de ces dernières années, des profits importants. La compétition redoublée sur leur marché traditionnel n'est sûrement pas étrangère à ce rapprochement, d'autant plus que certaines institutions de microfinance (IMF) ont bénéficié de méthodologies financières qui leur permettent de concurrencer les performances de crédits des banques traditionnelles, certaines se transformant même en «microbanques» réglementées et offrant également des facilités de microcrédits. Nous constatons donc un mouvement émergent de convergence des banques traditionnelles et des IMF sur le marché potentiellement profitable de la microfinance dont la demande est actuellement loin d'être couverte par les institutions financières en présence.

Des instruments innovateurs comme les systèmes de garantie peuvent contribuer au financement stable des IMF performantes et viables qui nécessitent un nantissement adéquat auprès des banques prêteuses, permettant à ces mêmes banques de se familiariser avec le secteur de la microfinance et aux IMF d'apprendre les exigences et les critères de performance des banques commerciales et de gagner en ultime instance une certaine crédibilité sur le marché des capitaux.

Dans ce contexte, l'expérience a démontré à Rafad que le succès d'un système de garantie dépend du bon respect d'un certain nombre de conditions ${ }^{18}$ :

- Une clarté dans la définition du destinataire du crédit.

- Un secteur financier qui ne souffre pas d'une liquidité insuffisante.

- Un système de garantie qui doit être crédible pour être efficace.

- Un système de garantie qui obtient un effet de levier financier grâce à la confiance de la banque commerciale, ce qui permet avec le temps un développement du crédit qui rend superflu le recours à ce même système de garantie.

17. Voir Annexe 1 : bibliographie sur les systèmes de garantie.

18. Las garantías bancarias, un instrumento financiero en debate, Dominique Lesaffre/Rafad, Folade 13 décembre 1997, Lima, Pérou, pp. 2-3. 
- Un système de garantie efficace visant l'autonomie financière, couvrant ses coûts opérationnels à moyen terme et ses pertes à long terme.

- Un système de garantie, qui, pour être durable, doit être attrayant, tant pour les bénéficiaires qui détermineront le volume de garanties, pour les banques commerciales qui agissent dans un cadre financier libéralisé avec des taux d'intérêts dictés par le marché, que pour les investisseurs qui financeront le fonds de garantie.

Tout en reconnaissant les difficultés rencontrées au cours des évaluations de systèmes de garantie,$^{19}$ il est utile de récapituler les principaux problèmes ${ }^{20}$ que des fonds de garantie ont rencontrés dans le passé:

- de la part des banques commerciales, on a pu constater parfois un comportement d' «oreiller de paresse», se traduisant par une étude superficielle du dossier de crédit, ou le risque «moral», laissant les propositions de crédit les plus risquées aux systèmes de garantie;

- de la part des emprunteurs: la baisse des taux de remboursement dès lors que le crédit est garanti (culture de crédit détériorée par le manque de discipline, en particulier lorsque le système est financé par la coopération occidentale);

- la difficulté de définir et de mesurer avec précision le rôle de la garantie: parle-t-on de volume de nouveaux prêts accordés en raison de la garantie ou de crédits de montants plus importants ou encore de taux d'intérêts inférieurs obtenus grâce à la garantie? Difficulté également de choisir les bons indicateurs permettant d'analyser et de mesurer le rôle joué par la garantie;

- l'expérience négative en général (inefficacité, mauvais résultats) en ce qui concerne les fonds de garanties publics qui sont souvent administrés de manière centralisée, bureaucratique et avec des objectifs fixes soumis à des restrictions de portefeuille;

- l'expérience négative de certains systèmes de garantie pour des crédits subventionnés ou de dépenses budgétaires du gouvernement, avec le risque de décapitalisation dans les cas de ressources budgétaires non assignées ou de forts taux de non-remboursement;

- la difficulté de pérenniser les systèmes de garantie qui restent encore souvent dépendants de subventions. Dans ce contexte, la pérennisation des systèmes de garantie doit s'analyser sur le long terme.

Il apparaît clairement à travers l'énumération des difficultés susmentionnées qu'il s'agit de systèmes de garantie qui sont souvent liés à l'utilisation de crédits subventionnés, gérés dans la plupart des cas par les banques elles-mêmes ou les services étatiques. Dans ces systèmes, les organisations locales, qui connaissent les particularités du secteur de l'économie populaire, et moins encore les acteurs économiques, ne sont impliquées dans la définition de la politique et l'application des systèmes de garantie.

19. Dues à: a) un manque de données quantitatives systématiques, b) un historique d'activités trop court, c) une absence d'études fondées vu les problèmes conceptuels et méthodologiques et les coûts élevés impliqués.

20. Las garantías bancarias, un instrumento financiero en debate, Dominique Lesaffre/Rafad, Folade, 13 décembre 1997, p. 4 
Parallèlement à ce système, on peut constater l'éclosion d'une deuxième génération de systèmes de garantie, dont le système Rafad fait partie. Ceux-ci donnent la préférence aux crédits qui suivent les taux d'intérêt du marché, travaillent étroitement avec les organisations liées à l'économie populaire, insistent sur la discipline dans le remboursement du crédit et sur la mobilisation de l'épargne qui servira de fonds de garantie local lors de la répartition du risque. Ces nouveaux systèmes mettent aussi l'accent sur l'importance du partenariat avec la banque locale et de son intérêt à explorer le secteur de la microfinance, afin de permettre un effet de levier. Dès lors, des leçons tirées des expériences avec les garanties bancaires dans le passé, découlent certaines règles à respecter pour assurer le succès de cette nouvelle génération de systèmes de garantie ${ }^{21}$.

Parmi les plus importants, notons les principes suivants:

- la garantie doit être limitée et perçue comme temporaire;

- la garantie doit être clairement définie, bien comprise et adaptée aux besoins spécifiques des bénéficiaires, résultat d'un engagement mutuel mûrement réfléchi et négocié entre garants, banques et IMF. En particulier, les prêteurs doivent considérer la garantie comme «attrayante» seulement dès lors qu'ils sont convaincus que le projet sous-tendant le crédit est économiquement viable et qu'ils feront - eux-mêmes - tout le nécessaire pour s'assurer du remboursement du crédit;

- les garanties nécessitent un suivi régulier permettant de mesurer de façon permanente la performance de l'IMF, en particulier quant aux taux de remboursement du prêt, le cash-flow, le déboursement des microcrédits;

- les garanties de type «intermédiaire» (comme l'exemple de «Nyesigiso») sont préférables à celle de type «individuel» ou de «portefeuille» (dossiers gérés par une ONG sans passer par une banque) ${ }^{22}$

- le succès de la garantie dépend de la capacité de gestion interne des IMF. Un renforcement institutionnel est indispensable, visant à améliorer leur système de gestion financière, la qualité de leur portefeuille et la gestion du risque;

- la documentation et la diffusion des «bonnes pratiques» en la matière sont essentielles au processus d'apprentissage tant des banques que des IMF;

- le succès ne devrait pas se mesurer seulement en terme de ressources financières additionnelles mobilisées, mais aussi en avantages indirects comme, par exemple, la confiance accrue des banques commerciales, une meilleure discipline financière des IMF, la transparence financière, etc.

- le rapprochement entre le secteur de la microfinance et le secteur bancaire ne dépendra pas seulement de la garantie, mais aussi de la volonté réelle et de l'intérêt de collaboration existant chez les banques ainsi que de la discipline et la transparence financière des IMF.

21. Contributions au séminaire sur le thème Systèmes de garantie dans un contexte de financement au développement, organisé par ADA les 7 et 8 septembre 1998, Mia Adams, ADA Dialogue, n 15, novembre 1998, pp. 7-10.

22. Voir dans la bibliographie les exemples de garanties accordées par: ACCION International, Women's World Banking, Appui au développement autonome (ADA Luxembourg). 


\section{ANNEXE: BIBLIOGRAPHIE SUR LES SYSTÈMES DE GARANTIE}

ACCION INTERNACIONAL. A Decade of Guaranteeing Succes. Accion Internacional Bulletin, vol. 30, no.1, Winter 1995.

ADA. Microentreprises, Actes du séminaire organisé à Munsbach les 25-26 au 26 juin. Luxembourg, 1996.

ADA. Microfinance, Actes du séminaire organisé le $1^{\text {er }}$ juillet à Luxembourg. Luxembourg, 1997.

Balkenhol et C. Lecointre. Les banques et la PME en Afrique de l'Ouest. Problèmes de financement: seize études de cas. Paris, L'Harmattan, 1996.

Bastiaenen, Michiel and Peter van Rooij. Guarantee Funds and NGOs: Promise and Pitfalls. A Review of the key Issues. Working paper no. 18. Geneva, ILO, 43p.

Baydas, Mayada., and others. Commercial Banks in Micro-finance: New Actors in the Microfinance World. Microenterprise Best Practices 1997.

Brugger, Ernst A., and others. Impact of the FUNDES guarantee programs on small businesses in Latin America, 1996.

Castellanos, Jorge. "The Financial Supervision of Loan Guarantors". The Financier, vol. 4 Issue 1 \& 2 Feb-May 1997, pp. 34-43.

CGAP. Microcredit Interest Rates. Occasional Papers, 1996.

Craig, Churchill. Regulation and Supervision of Microfinance Institutions: Case Studies, The microfinance Network. Occasional Paper no 2. 1997.

Credit Guarantees Bibliography. Devfinance, Ohio University, 1998.

Demdoum, Samira. Les garanties bancaires: outils de développement et de contrôle des ONG. Mémoire de fin d'études. Université de Mons-Hainaut, 1997, 80 p.

Diouf, M. La coopération: une nécessité entre les banques et les opérateurs de la micro-finance. ADA Dialogue n 8 pp. 29-25.

Ditchter, T.W. Questioning the Future of NGO'S in Microfinance. ADA Dialogue no.11, pp. 23-28.

Gallardo, Joselito and others. A commercial bank's micro-finance programs the case of Hatton National Bank in Sri Lanka. World Bank, Discussion paper, 369 p.

Garson, José. Microfinance and anti-poverty strategies. Occasional Papers, New-York, 1996.

Gilbert, Pierre Luigi. "Pricing Credit Risk in Loans and Guarantees". The Financier, vol. 3 Issue 4/5 Nov-Dec. 1996, pp. 26-32.

Graham Bannock and Partners, Ltd. Credit Guarantee Schemes for Small Business Lending: A Global Perspective, vol. 1 - Main Report, April 1997.

Gudger, Michael The sustainability of credit guarantee systems, Small Enterprise Development, vol. 8, no. 2, June 1997.

Gudger, Michael. Credit Guarantees: An Assessment of the State of Knowledge and new Avenues of Research, FAO, (November 1997, Draft).

Hatakeyama, Michiko, S. and others. Credit Guarantee systems for Small and Medium Enterprise in Some Asian Countries. The Financier, vol. 4 Issue 1 \& 2, Feb-May 1997, pp. 67-23.

Kaboré, Noël. Relations between Conventional Agricultural Banking and Decentralized Financial Systems: Two Approaches. Current situation and outlook in Burkina Faso. Techniques financières et développement, Special Supplement, 1997, pp. 21-26

Le risque dans l'attribution de garanties aux organisations de développement du secteur non formel du tiers monde. Edité par Fernand Vincent, Rafad, Gèneve, 1995, 59 p.

Levistsky, Jacob. Credit Guarantee Funds and Mutual Guarantee Systems? Small Enterprise Development, vol. 4 no 2, June 1993.

Levitsky, Jacob and Prasad Ranga N. Credit Guarantee Schemes for Small and Medium Enterprises, The Financier, vol. 4 Issue 1 \& 2, Feb-May 1997, pp. 62-73.

Llorens, Juan Luis. Loan Guarantee System for SMEs in Europe. The Financier, vol. 4 Issue 1 \& 2, FebMay 1997, pp. 74-85.

Malhotra, Mohini. "Microfinance: The New Emerging Market ?" CGAP, 1997.

Marulande de García, Beatriz. National Guarantee Fund of Colombia. The Financier, vol. 4 Issue 1 \& 2 Feb-May 1997, pp. 44-50.

McGuire, Paul B. and John D. Conroy. Bank-NGO Linkages and the transaction costs to the lending to the poor through groups, Small Enterprise Development, vol. 8 no 1, pp. 4-15.

Meyer, Richard L. and Nagarajan Geetha. Credit Guarantee Schemes for Developing Countries: Theory, Design and Evaluation Center for Economic Growth, Ohio University, 1996.

Mosley, Paul. Metamorphosis from NGO to Commercial Bank: the case of Bancosol in Bolivia. Institute for Developpement Policy and Management, Working Paper, no 4, 1993, 45 p. 
Oehring, Eckart. The FUNDES Experience with Guarantee Systems in Latin America: Model, Results and Prospects. The Financier, vol. 4 Issue 1 \& 2 Feb-may 1997, pp. 57-61.

Otero, Maria. Accessing Commercial Capital for Microfinance: The ACCION Experience. Speech delivered at a seminar on Rural Finance at the World Bank, October 24, 1996.

Pomareda, C. and Max Soto. Regulación Organizaciones Financieras No Convencionales y Microfinanciamiento: estado de la discusión. Folade, Costa Rica, 1977.

Round Table on Credit Guarantee Systems: International Experiences and Lessons for Latin American and the Caribbean. Inter-American Development Bank, Washington D.C., June 27-28, 1996.

Rowlatt, Amanda. Microfinance - Involvement Banks, Small Enterprise Development, vol. 8, no 2, 1997.

Shari Berenbach and Craig Churchill. Risk Profile and Regulatory Approaches to Micro-finance Institutions, in Regulation and Supervision Microfinance Institutions. Experience from Latin America, Asia and Africa. The Microfinance Network, Occasional Paper no 1, pp. 19-30.

Smith, Anne-Marie. Bank Dagang Bali: A Profitable Commercial Bank in Microfinance. CGAP, 1977.

Stearns, Katherine. Leverage or Loss Guarantee Funds and Microenterprise, ACCION International, Monograph Series no 8.

Thembani International Guarantee Fund (TIGF): Banking on South Africa's Democratic Development. Johannesburg, TIGF Office, 1996.

Vogel, Robert C. and Dale W. Adams. Cost and Benefits of Loan Guarantee Programs. The Financier, vol. 4 Issue 1 \& 2 Feb-May 1997, pp. 22-29.

Vogel, Robert C. and Dale W. Adams. Rationale for Establishing Credit Guarantee Systems, Paper Presented at the Round Table on Credit Guarantee Systems: International Experiences and Lessons for Latin America and the Caribbean, Inter-American Development Bank, Washington D.C. June 27-28, 1996, 15 p.

Women's World Banking. The Missing Links: Financial Systems that Work for the Majority. WWB, 1995,38 p.

Young, Robin; Lara Goldmark, and others. Microfinance Guarantees: A Basic Primer and Review of Experiences in Latin America and the Caribbean, Inter-American Development Bank, Microenterprise Unit, December, 1997, 29 p. 